\title{
3D TRANSIENT THERMAL MODELLING OF LASER MICRO-CHANNEL FABRICATION IN LIME-SODA GLASS
}

\author{
A. Issa, D. Brabazon and M. S. J. Hashmi \\ School of Mechanical and Manufacturing Engineering, Dublin City University, Ireland, \\ email: ahmed.issa2@mail.dcu.ie; dermot.brabazon@dcu.ie
}

\begin{abstract}
Laser-fabricated microchannels in glass offer a wide range of bioengineering and telecommunication applications. A $1.5 \mathrm{~kW} \mathrm{CO}$ laser with $10.6 \mu \mathrm{m}$ wavelength was used in this study to fabricate micorchannels on the surface of soda-lime glass sheets. A thermal model of the process was developed based on transient heat conduction due to a pulsed heat input. The resulting equation predicted the temperature distribution in the regions surrounding the laser focus. Temperature - time curves were drawn from those equations, which were useful in estimating the thermal history in the processed samples. The temperature distribution was also used to predict the channel geometry (based on the vaporisation temperature of glass). Most of the laser power used was consumed in bringing the glass to the vaporisation temperature. The model was able to predict the channel width, depth and surface roughness. These laser-fabricated channel characteristics were measured and compared to the results obtained from the thermal model. The laser power, frequency, pulse width and translation speed were the control parameters in both studies; hence a direct comparison was established between the model and the experimental results.
\end{abstract}

\section{KEYWORDS: $\mathrm{CO}_{2}$ laser, glass, microchannels, thermal modelling}

\section{INTRODUCTION}

Glasses absorb laser radiation in a way that depends highly on the incident wavelength [1, 2], most transparent materials are opaque in the visible region but absorb strongly at or near 10 $\mu \mathrm{m}$, which makes $\mathrm{CO}_{2}$ laser very efficient for machining these materials [1,3]. Laser-induced plasma will also enhance the coupling of laser radiation to the material's surface raising its temperature to the vaporising temperature at about $3500^{\circ} \mathrm{C}$ [4], which is sufficient to ablate the material from the irradiated region. This can be accomplished despite the fact that glasses may in some cases reflect up to $30 \%$ of the incident laser radiation [3]. Micro-channel fabrication, on the surface or in the bulk of glass sheets is widely used in various applications such as telecommunication, optical and bio-medical engineering [5, 6]. Some studies [7, 8] have investigated irradiating glass samples repeatedly along the same path to investigate the effect of the amount of radiation deposition on the microchannel fabrication. However, such studies use mainly fixed operating parameters.

If thermal modelling of the laser heat deposition is used, then it is possible to estimate the resulting effects on the materials $[1,9]$. It can be useful to estimate the temperature ranges and thermal stresses that the material is expected to experience beforehand. This study covered the modelling and the production of single microchannels. However, the concepts and results presented are extendible to cover other 2D and 3D microchannels. The model and principles 
presented in this paper are also applicable to metallic material processing and heat treatment using pulsed laser irradiation.

\section{THE THERMAL MODEL}

Heat conduction problems in a medium can be expressed in terms of partial differential equations of temperature with respect to space and time. The general form of the heat conduction equation, provided that there is no heat generation in the medium and that all the thermal properties are constants [10]:

$\rho c_{p} \frac{\partial T}{\partial t}=k\left(\frac{\partial^{2} T}{\partial x^{2}}+\frac{\partial^{2} T}{\partial y^{2}}+\frac{\partial^{2} T}{\partial z^{2}}\right)$

where, $\rho\left(\mathrm{kg} / \mathrm{m}^{3}\right), c_{p}\left(\mathrm{~J} / \mathrm{kg} .{ }^{\circ} \mathrm{C}\right), k\left(\mathrm{~W} / \mathrm{m} .{ }^{\circ} \mathrm{C}\right)$ are the density, heat capacity and thermal conductivity of the material respectively. If the medium is moving with a constant velocity $U$ parallel to the X-axis, as shown in figure 1, then equation (1) becomes [10]:

$\frac{\partial T}{\partial t}+U \frac{\partial T}{\partial x}=\alpha\left(\frac{\partial^{2} T}{\partial x^{2}}+\frac{\partial^{2} T}{\partial y^{2}}+\frac{\partial^{2} T}{\partial z^{2}}\right)$

where, $\alpha\left(\mathrm{m}^{2} / \mathrm{s}\right)$ is the thermal diffusivity of the material, equal to $k / \rho c_{p}$.

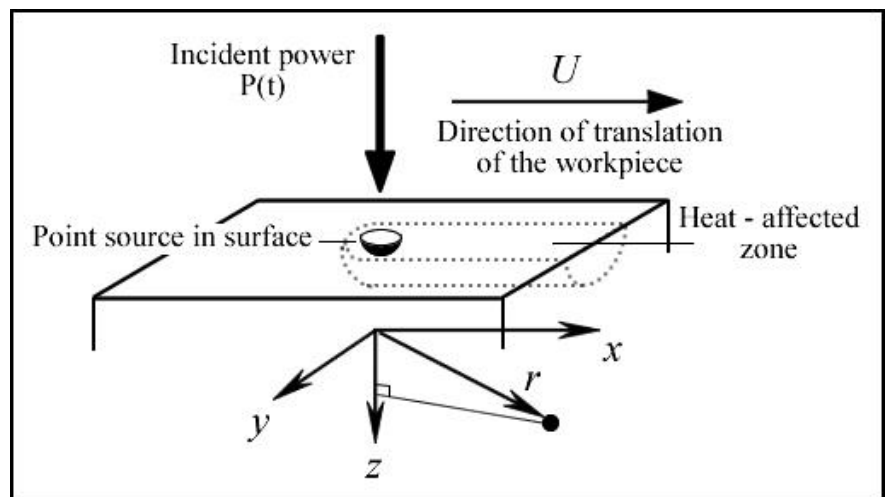

Figure 1: Heat point source in the surface of the workpiece.

Solving equation (2) gives a transient heat or temperature distribution in the medium in the form $\mathrm{T}(\mathrm{x}, \mathrm{y}, \mathrm{z}, \mathrm{t})$. However, the solution is not unique to all cases and depends on a number of assumptions, initial conditions and boundary conditions. The assumptions made in this work for the solution are:

1- The initial temperature is equal to the ambient temperature $\mathrm{T}(\mathrm{x}, \mathrm{y}, \mathrm{z}, 0)=\mathrm{To}$.

2- The medium is a slab of two parallel planes and heat enters from one plane at $\mathrm{z}=0$. This solution is a heat conduction one, it requires that there is no heat losses from the medium at its two planes, i.e. $\partial T / \partial z=0$.

3- There are no phase changes in the medium. Although the solution does not explicitly include the latent heat effects, it is still useful in estimating the penetration of the melting isotherms under the conditions of melting via conduction [1].

4- The medium is a semi-infinite solid, meaning that the temperature variations in the region of interest do not affect the temperatures in regions considered to be far a way from it, such as the 
bottom plane. This assumption has been verified by applying the same conditions on different thicknesses and getting the same result.

Based on these assumptions, equation (2) can be solved for a pulsed laser source, with a pulse repetition frequency $(\mathrm{PRF})$ given in pulses per second $(\mathrm{Hz})$. The incident laser beam is modelled as a time-dependent point heat source at $(0,0,0)$. The solution of equation $(2)$, shown in equation (3), is based on the impingement of temperature harmonics on a slab of material $[9,10]$.

$T(x, y, z, t)=T_{o}+\frac{P(t)}{2 \pi k r} \exp \left[i \omega t+\frac{U}{2 \alpha}\left(x-r \sqrt{1+\frac{4 \alpha \omega i}{U^{2}}}\right)\right]$

where, $P(t)$ is the time dependent power input, $\omega$ is the fundamental frequency of the power input $=2 \pi$.PRF, and $r$ is the radial distance from the power source $=\sqrt{x^{2}+y^{2}+z^{2}}$.

The square root taken in equation (3) must be the positive real part. A pulsed laser power input as the one shown in figure 2 is to be used. This power source has a period $t_{0}=1 / \mathrm{PRF}$ and is given in the interval $-1 / 2 \mathrm{t}_{\mathrm{o}} \leq \mathrm{t}<1 / 2 \mathrm{t}_{\mathrm{o}}$ by

$\mathrm{P}(\mathrm{t})= \begin{cases}\mathrm{P}_{\mathrm{o}} \mathrm{t}_{\mathrm{o}} / \tau & |\mathrm{t}|<1 / 2 \tau \\ 0 & 1 / 2 \tau<|\mathrm{t}|<1 / 2 \mathrm{t}_{\mathrm{o}} \\ \mathrm{P}_{\mathrm{o}} \mathrm{t}_{\mathrm{o}} / 2 \tau & \text { otherwise }\end{cases}$

where, $\mathrm{P}_{\mathrm{o}}(\mathrm{W})$ is the average power and $\tau(\mathrm{s})$ is the pulse width.

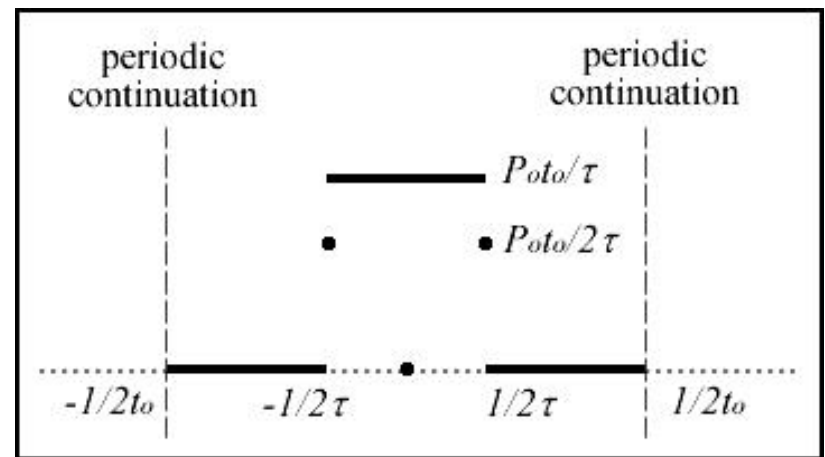

Figure 2: The pulsed power input given by equation (4).

Then it is possible to express this power signal in terms of a Fourier series of the form

$P(t)=P_{o}+\sum_{n=1}^{\infty} a_{n} \cos \frac{2 \pi n t}{t_{o}}=P_{o}+\sum_{n=1}^{\infty} a_{n} \operatorname{Re}\left(\exp \frac{2 \pi n t}{t_{o}} i\right)$

in which,

$a_{n}=\frac{2}{t_{o}} \int_{-\tau / 2}^{\tau / 2} \frac{P_{o} t_{o}}{\tau} \cos \frac{2 \pi n t}{t_{o}} d t=\frac{2 P_{o} t_{o}}{t_{o} \tau} \int_{-\tau / 2}^{\tau / 2} \cos \frac{2 \pi n t}{t_{o}} d t=\frac{2 P_{o}}{\tau} \times \frac{t_{o}}{2 \pi n} \times\left.\sin \frac{2 \pi n t}{t_{o}}\right|_{-\tau / 2} ^{\tau / 2}$

$=\frac{P_{o} t_{o}}{\pi n \tau}\left(\sin \frac{2 \pi n \tau}{2 t_{o}}-\sin \frac{-2 \pi n \tau}{2 t_{o}}\right)=\frac{2 P_{o} t_{o}}{\pi n \tau} \sin \frac{\pi n \tau}{t_{o}}$

thus, 
$P(t)=P_{o}+\frac{2 P_{o} t_{o}}{\pi \tau} \sum_{n=1}^{\infty} \frac{1}{n} \sin \frac{n \pi \tau}{t_{o}} \cdot \operatorname{Re}\left(\exp \frac{2 \pi n t}{t_{o}} i\right)$

Substituting 5 into 3 gives

$$
\begin{aligned}
T(x, y, z, t)= & T_{o}+\frac{P_{o}}{2 \pi k r} \exp \left[\frac{U}{2 \alpha}(x-r)\right]+ \\
& +\frac{P_{o} t_{o}}{\pi^{2} k r \tau} \sum_{n=1}^{\infty} \frac{1}{n} \sin \frac{n \pi \tau}{t_{o}} \operatorname{Re}\left(\exp \left[\frac{2 n \pi t}{t_{o}} i+\frac{U}{2 \alpha}(x-r \sqrt{1+i n \mathrm{Si}})\right]\right)
\end{aligned}
$$

which when taking the real parts of the exponential and the square root gives

$$
\begin{aligned}
T(x, y, z, t)=T_{o}+\frac{P_{o}}{2 \pi k r} \exp \left[\frac{U}{2 \alpha}(x-r)\right]+ & \\
+\frac{P_{o} t_{o}}{\pi^{2} k r \tau} \sum_{n=1}^{\infty} \frac{1}{n} \sin \frac{n \pi \tau}{t_{o}} \exp \left[\frac{U}{2 \alpha}(x-r \mathrm{Sn})\right] \times & \\
& \times \cos \left(\frac{2 n \pi t}{t_{o}}-\frac{U r n \mathrm{Si}}{4 \alpha \mathrm{Sn}}\right)
\end{aligned}
$$

where, $\mathrm{Si}=(8 \pi \alpha) /\left(t_{o} U^{2}\right)$, is Simon's number that characterises a periodic solution with period $\mathrm{t}_{\mathrm{o}}$ and was substituted in equation (3), and $\mathrm{Sn}=\sqrt{\left[1+\sqrt{1+n^{2} \mathrm{Si}^{2}}\right] / 2}$.

Other methods of obtaining solutions similar to equation (6) are given using Fourier series and Laplace transform [10]. Equation (6) was used to calculate the temperature distribution in the workpiece, $\mathrm{T}(\mathrm{x}, \mathrm{y}, \mathrm{z}, \mathrm{t})$, based on the power, PRF, pulse width and sample material translational speed as the process parameters.

\section{EXPERIMENTAL WORK}

Two millimetre thick commercial soda-lime glass sheets were used in this work. A $1.5 \mathrm{~kW}$ $\mathrm{CO}_{2}$ laser beam was focused using a $127 \mathrm{~mm}$ focal length lens to a spot diameter of approximately $90 \mu \mathrm{m}$ on the surface of the samples and delivered via a nozzle with an air jet at one bar. Channels of $15 \mathrm{~mm}$ in length were produced. The laser power (P), pulse repetition frequency (PRF), pulse width $(\tau)$ and scanning speed $(\mathrm{U})$ were chosen as the process control parameters. After producing the channels the samples were later on the same day water washed and air jet dried. A gold coating was then applied in order to facilitate laser scanning of the samples. A three dimensional profile for each channel was generated by scanning a $50 \mu \mathrm{m}$ length section of each channel at a resolution of $1.95 \mu \mathrm{m}$ in the lateral directions ( $\mathrm{x}$ and $\mathrm{y}$ ) and $0.5 \mu \mathrm{m}$ in the height direction (z) [11]. Each channel was scanned at the same position, $10 \mathrm{~mm}$ from the start of the channel. The channel parameters measured from these profiles were chosen to be the width $(\mu \mathrm{m})$, the depth $(\mu \mathrm{m})$ and the surface roughness, $\mathrm{Ra},(\mu \mathrm{m})$. Three measurements were made and averaged for each parameter. The surface roughness (Ra), taken along the same direction as the channel axis, was measured along the bottom of the channel and half way up either side of the channel. The Ra measurement can be particularly significant in fluid flow and 
wave guide applications. All measurements were performed using a software code built specifically for this study.

\subsection{Process considerations}

The weight percentage of soda-lime components used in this work were taken as $73 \% \mathrm{SiO}_{2}$ $-15 \% \mathrm{Na}_{2} \mathrm{O}-7 \% \mathrm{CaO}-4 \% \mathrm{MgO}-1 \% \mathrm{Al}_{2} \mathrm{O}_{3}$. The density was taken as $\rho=2500 \mathrm{~kg} / \mathrm{m}^{3}$, the heat capacity was taken as $C_{p}=870 \mathrm{~J} / \mathrm{kg} .{ }^{\circ} \mathrm{C}$ and the thermal conductivity as $k=1.06 \mathrm{~W} / \mathrm{m} .{ }^{\circ} \mathrm{C}$, which give a thermal diffusivity value of $\alpha=4.8736 \times 10^{-7} \mathrm{~m}^{2} / \mathrm{s}$ [2]. Based on the laser intensity range used in this work $\left(0.3-0.5 \mathrm{MW} / \mathrm{cm}^{2}\right)$, the plasma formed is a laser-supported combustion plasma (LSC). During the initial stages of the pulse, LSC plasma starts to form, shielding up to $90 \%$ the incident power from the target [4]. However, as the plasma begins to expand radially, the coupled energy increases to $50 \%$ [4, 12]. The radiative losses in this plasma scheme are typically 15 to $20 \%$ of the incident power. There is a significant amount of energy consumed to raise the glass to its melting and consequently to its vaporisation temperature. These amounts of energies are called the latent heat of melting and vaporisation, respectively. Simple calculations can show the amount of energy needed to raise the focal volume to the vaporisation temperature. The amount of heat required to reach the vaporisation temperature, $\mathrm{T}_{\mathrm{v}}$, equals $C_{p} \cdot\left(\mathrm{T}_{\mathrm{v}}-\mathrm{T}_{\mathrm{o}}\right)=$ $870 .(3427-22)=2,962,350 \mathrm{~J} / \mathrm{kg}$. The amount of heat required to raise the temperature of the vapour to approximately 10 times the vaporisation temperature, $\mathrm{T}_{\mathrm{s}}$, was also calculated. This allowed an approximation of the energy required to ablate the material in order to form the channel, as per the procedure followed by other workers [4]. This energy was calculated as $C_{p} \cdot\left(\mathrm{T}_{\mathrm{s}}-\mathrm{T}_{\mathrm{v}}\right)=870 .(34270-3427)=26,833,410 \mathrm{~J} / \mathrm{kg}$. The scale length of diffusion was obtained from, $L_{D}=\sqrt{\alpha \cdot \tau}$, where $\alpha$ and $\tau$ are the thermal diffusivity and the laser pulse width. The average pulse width used in this work was $1.53125 \mathrm{~ms}$, so that $\mathrm{L}_{\mathrm{D}}=27.32 \mu \mathrm{m}$ was the average depth affected by the heat wave diffused per a single pulse. The volumetric mass that will be affected by this heat wave can be calculated from, $m=\rho \cdot \pi \cdot r^{2} \cdot L_{D}$, where $\mathrm{r}$ is the laser beam's radius. The average heated mass, $\mathrm{m}$, was equal to $4.345 \times 10^{-10} \mathrm{~kg}$. This mass was multiplied by the two latent heat of diffusion values calculated above, which gave the amount of energies required to bring this irradiated volume to its vaporisation temperature and then to being ablated. These were $1.287 \mathrm{~mJ}$ for heating the volume to its vaporisation temperature and $11.659 \mathrm{~mJ}$ for ablation.

In this study the laser beam's power, $\mathrm{P}$, was varied between 18 and $30 \mathrm{~W}$, and the pulse repetition frequency, PRF, was varied between 160 and $400 \mathrm{~Hz}$, this resulted in minimum and maximum pulse energies of 45 and $187.5 \mathrm{~mJ}$ respectively. Table 1 lists the minimum and maximum pulse energies produced in this study and the minimum percentages of these needed for breakdown.

Table 1: Relative breakdown energies.

\begin{tabular}{|l|l|l|l|}
\hline Expt. energy $(\mathrm{mJ})$ & \% For heating & \% For ablating & \% Total \\
\hline 45 & 2.86 & 25.91 & 28.77 \\
\hline 187.5 & 0.69 & 6.22 & 6.91 \\
\hline
\end{tabular}

The breakdown threshold energy, $E_{t h}$, was observed at $45 \mathrm{~mJ}$, which corresponded to a fluence, $\phi_{t h}=E_{t h} / \pi \cdot r^{2}=707.55 \mathrm{~J} / \mathrm{cm}^{2}$. A channel that was used to identify the breakdown threshold in this work is shown in figure 3 (a). The surface cracks in this figure were produced using $\mathrm{P}=18 \mathrm{~W}, \mathrm{PRF}=400 \mathrm{~Hz}$ and $\mathrm{U}=8.333 \mathrm{~mm} / \mathrm{s}$, which corresponds to the first row in table 1. The structural changes in this image suggest that about $28 \%$ of the incident power was 
necessary to initiate ablation; the remainder of the power was shielded by the induced plasma. At higher fluences, wider and deeper structural changes, as shown in figure 3 (b), were produced. This channel was produced using $\mathrm{P}=30 \mathrm{~W}, \mathrm{PRF}=160 \mathrm{~Hz}$ and $\mathrm{U}=8.333 \mathrm{~mm} / \mathrm{s}$. These settings correspond to the second row in table 1 . The larger dimensions in the channel in figure 3 (b) suggest that more than $6.91 \%$ of the energy was coupled to the sample. The larger pulse duration from the processing parameters to produce the channel in figure 3 (b) would be expected to allow time for the heat to diffuse and further energy to be coupled to the target $[4,12]$.

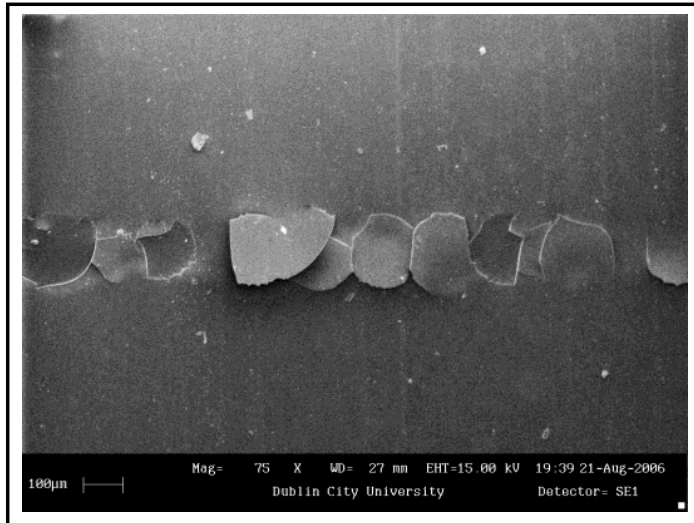

(a)

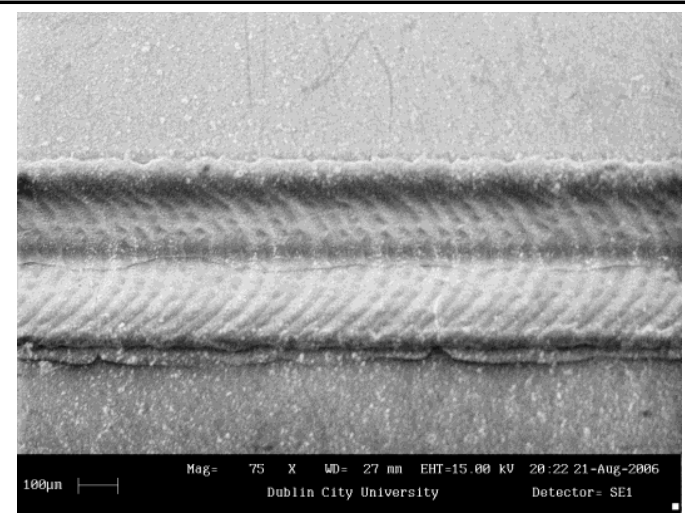

(b)

Figure 3: SEM image of channels produced at (a) minimum and (b) maximum fluence settings.

Based on the total percentages in table 1, plasma shielding theory, radiative losses associated with the LSC plasma scheme, and simulation trial and error procedures on experimental data, an average $25 \%$ of the incident beam's power was calculated as being delivered to the sample. This $25 \%$ of the incident power was used for solving the thermal mathematical model. Based on the pulse energy range shown in table 1, the mathematical model would in some cases, over-estimate or under-estimate the channel dimensions. This would not affect the predicted channel shape; however, it has implicitly reduced the complexity of analysis compared to taking phase changes into account in the mathematical model [1].

\subsection{Method of calculations}

All the calculations and graphical displays were performed with a number software codes built for this study. To solve equation (6), the laser power source was assumed to be at $(0,0,0)$ for all instances of time. Since the workpiece is translated in the $\mathrm{x}$ direction at a constant velocity of $U$, the $x$ values change with time based on a Eularian coordinate, according to $x=a+t^{*} U$, where, $a$, is the initial $x$ location and, $t$, is the time elapsed since the beginning of translation [9].

\subsubsection{Planar isotherms}

For each z-plane, the time and the y instances were needed to solve equation (6). Hence, a $2 \mathrm{D}$ mesh in time and space $(\mathrm{t}, \mathrm{y})$ was constructed. The first element is the pulse duration, $\mathrm{t}_{\mathrm{o}}=$ $1 / \mathrm{PRF}$, which also corresponds to the $\mathrm{x}$-axis values, as mentioned earlier. The second element of the mesh was a sufficiently large value of y to cover half of the channel width, since the solution is symmetric about the $y$-axis, this also saved analysis time. Solving for a constant value of $z$ and setting $\mathrm{x}=0$ when $\mathrm{t}=0$ produced the isotherms in that plane. The solution value at each mesh point was independent of the mesh size. Figure 4 shows the predicted isotherms of three pulses at plane $\mathrm{z}=0$ using $\mathrm{P}=24 \mathrm{~W}, \mathrm{PRF}=228 \mathrm{~Hz}, \tau=1.53125 \mathrm{~ms}$, and $\mathrm{U}=5 \mathrm{~mm} / \mathrm{s}$. The mesh was generated for $\mathrm{t}=0$ to $\mathrm{t}_{\mathrm{o}}=0-1 / 228=(0-4.375 \mathrm{~ms})$ and $\mathrm{y}=0$ to $100 \mu \mathrm{m}$. Iteration step sizes in 
the $\mathrm{x}$-axis were $0.37 \mu \mathrm{m}$. The step sizes in the $\mathrm{y}$-axis were $1 \mu \mathrm{m}$. The resulting isotherm was reflected about the $y$-axis to give the full channel width. The solution is periodic in time, so that solving for one pulse duration, $t_{0}$, was sufficient to calculate the thermal profile information which could then be repeated for the proceeding pulses. The temperature tended to infinity as y approached 0 , so for this particular simulation the temperatures were truncated at $20,000{ }^{\circ} \mathrm{C}$. The data cursors on the isotherms in figure 4 show details of location and temperature $(\mathrm{x}, \mathrm{y}, \mathrm{T})$ at two points. These points are of particular importance as they are on the isotherm of vaporisation at a temperature around $3500{ }^{\circ} \mathrm{C}$.

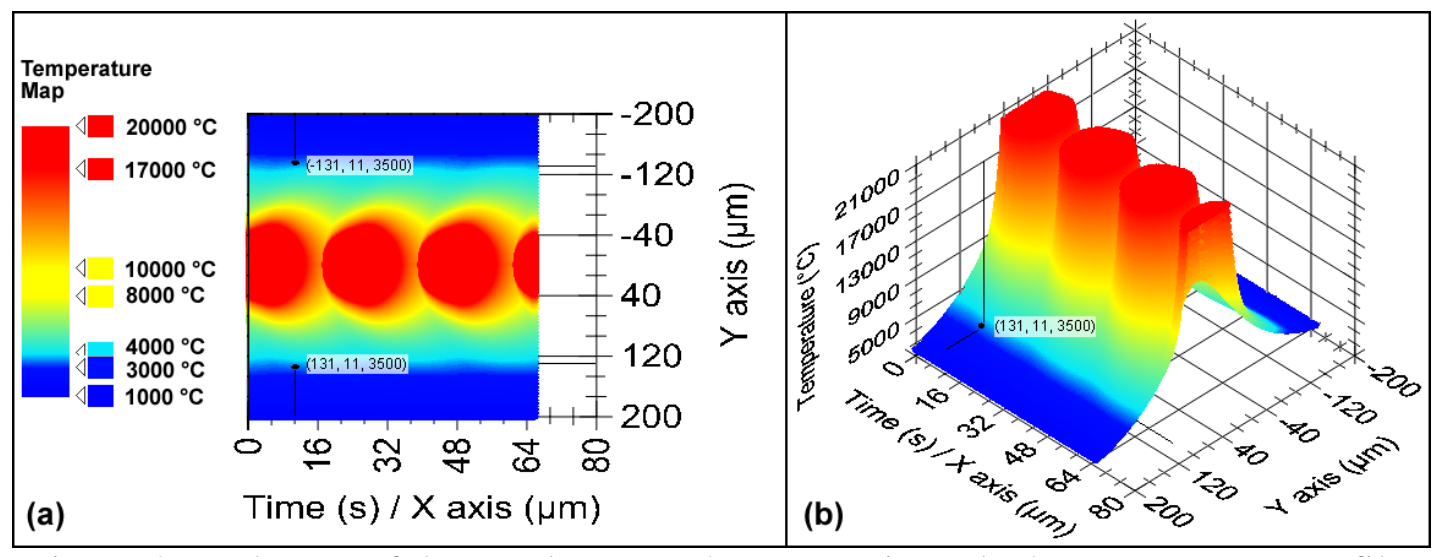

Figure 4: Isotherms of three pulses at $\mathrm{z}=0$, (a) top view, (b) 3D temperature profile.

\subsubsection{Thermal history}

Equation (6) can also be solved to predict the thermal history of specific points in the plane of interest. This can be useful in monitoring how the temperatures change at those points over a period of time which exceeds the pulse duration and enables the study the heating and cooling rates which can be useful in estimating the resulting microstructures due to laser irradiation. Figure 5 shows the thermal histories of the same sample as in figure 4 , for four points at $y=10$, 150, 500 and $1000 \mu \mathrm{m}$. The initial x coordinate was set so that it crossed the laser point source at $\mathrm{t}=0.2$ seconds. The temperatures were calculated for a total time of 2 seconds so at the traversing speed of $5000 \mu \mathrm{m} / \mathrm{s}$ the y points were a perpendicular distance of $9000 \mu \mathrm{m}$ behind the laser point source.

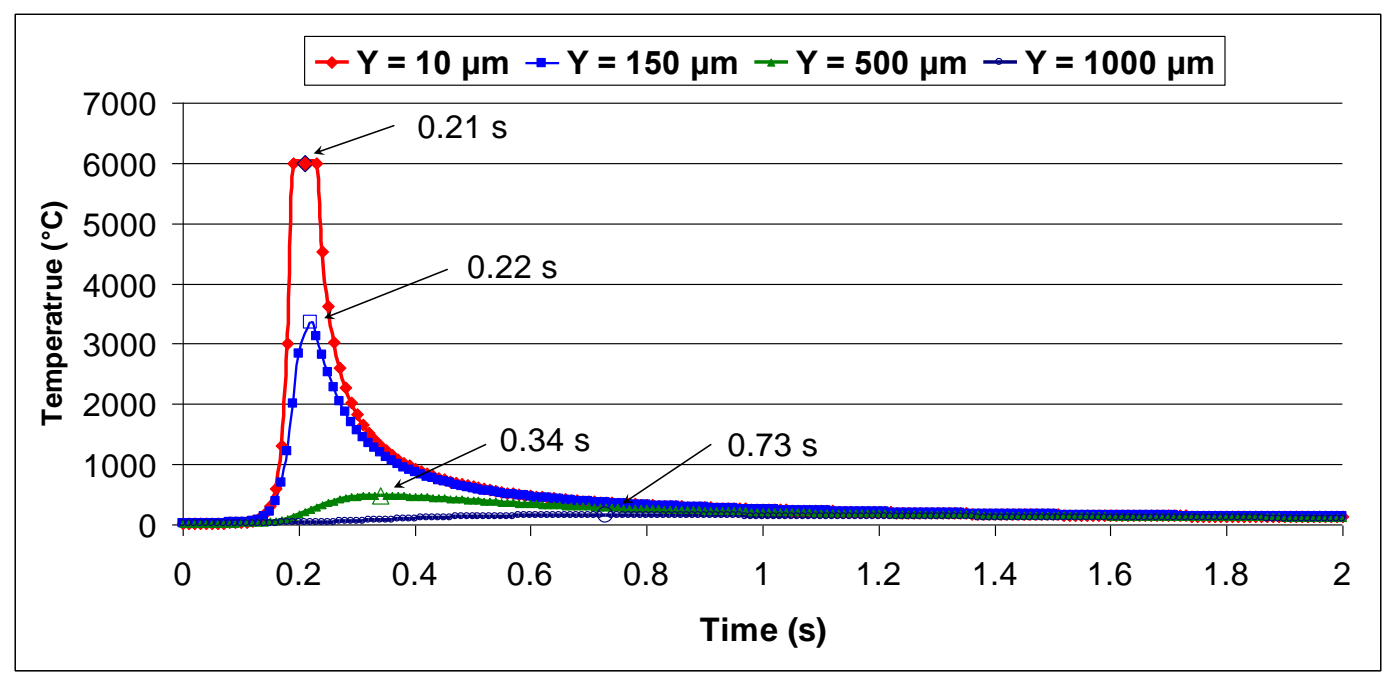

Figure 5: Thermal histories of 4 points on the surface of glass sheet. 
The maximum temperature at point $\mathrm{y}=10 \mu \mathrm{m}$ occurred at the truncated $6,000{ }^{\circ} \mathrm{C}$. The maximum temperatures were reached at $0.21,0.22,0.34$ and 0.73 seconds for the points $\mathrm{y}=10$, 150,500 and $1000 \mu \mathrm{m}$, respectively. This indicates that substantial time duration is required for the heat to be diffused as we move far from the heat source. The temperature falls below $3500{ }^{\circ} \mathrm{C}$ as the $\mathrm{y}$ value becomes more than $130-150 \mu \mathrm{m}$. This can also be noted from the isotherms in figure 4 . It can also be noted that the cooling rates are different for the four points in figure 5, this may be used to predict the resulting microstructure in those regions.

\subsubsection{Channel 3D geometry}

As explained earlier in 3.2.1, the model calculation procedure was repeated for all values of $\mathrm{z}$ until the maximum levels of temperature fall below a desired value, usually $\pm T_{\mathrm{v}}$ so that the channel geometry can be predicted. The geometry of the channel was predicted by obtaining the $\mathrm{x}, \mathrm{y}$ and $\mathrm{z}$ coordinates at which the temperatures were at $\mathrm{T}_{\mathrm{v}}$ from planar isotherms spaced approximately $1 \mu \mathrm{m}$ apart and ranged between $\mathrm{z}=0$ and the depth of the channel. The channel width, depth and surface roughness were also calculated from these predicted geometries using the same procedure performed on the experimental scans.

\section{Results and comparison}

The predicted values for channel width, depth and roughness are listed in table 2 with the corresponding experimental values. The results shown in table 2 were selected from a design of experiments that covered the range of processing parameters examined in another study [13]. These experimental values were taken to validate and compare them with the predicted values from the thermal mathematical model.The effects of P, PRF and U were studied on the resulting width and depth of the channels [14]. It was found that the P had a direct proportional effect, while PRF and $U$ had an inverse proportional effect on the channel dimensions.

Table 2: List of microchannel processing parameters including model and experimental values for channel width, depth and roughness.

\begin{tabular}{|c|c|c|c|c|c|c|c|c|}
\hline $\begin{array}{l}\text { Ch. } \\
\text { No. }\end{array}$ & $\begin{array}{c}\mathbf{P} \\
(\mathbf{W})\end{array}$ & $\begin{array}{l}\text { PRF } \\
(\mathrm{Hz})\end{array}$ & $\tau(\mathrm{ms})$ & $\mathbf{U}(\mathbf{m m} / \mathbf{s})$ & & $\begin{array}{c}\text { Width } \\
(\mu \mathrm{m})\end{array}$ & $\begin{array}{c}\text { Depth } \\
(\mu \mathrm{m})\end{array}$ & $\mathbf{R a}(\mu \mathbf{m})$ \\
\hline \multirow{3}{*}{1} & \multirow{3}{*}{18} & \multirow{3}{*}{160} & \multirow{3}{*}{3.125} & \multirow{3}{*}{8.33} & Model & 192.1 & 96.2 & 7.229 \\
\hline & & & & & Experiment & 302 & 74 & 3.848 \\
\hline & & & & & \% Difference & 36.39 & -30 & -87.86 \\
\hline \multirow{3}{*}{2} & \multirow{3}{*}{24} & \multirow{3}{*}{228} & \multirow{3}{*}{1.531} & \multirow{3}{*}{5} & Model & 265.9 & 132.98 & 3.615 \\
\hline & & & & & Experiment & 267 & 148 & 4.495 \\
\hline & & & & & \% Difference & 0.41 & 10.14 & 19.58 \\
\hline \multirow{3}{*}{3} & \multirow{3}{*}{30} & \multirow{3}{*}{400} & \multirow{3}{*}{0.5} & \multirow{3}{*}{5} & Model & 299.12 & 144.48 & 1.047 \\
\hline & & & & & Experiment & 308 & 142 & 3.515 \\
\hline & & & & & \% Difference & 2.88 & -1.75 & 70.21 \\
\hline
\end{tabular}

\subsection{Channel parameters}

The maximum error in width prediction was $36.39 \%$ in the case of channel 1 . The maximum depth prediction error was $-30 \%$ for channel 1 . Having the highest pulse energy, channel 1 prediction error agrees with the theory, outlined earlier that, at higher fluences, the delivered energy is larger than $25 \%$ of the incident laser beam. Furthermore on this case, both width and depth errors are in agreement indicating that the model is under-estimating the actual results. These prediction errors have not affected the accuracy of shape prediction as explained in the next section. The errors in roughness prediction are significantly high due to the small scale of the parameter being measured. Channel roughness values obtained from this study were in the 
range of 2 to $12 \mu \mathrm{m}$, and in reality this parameter depended on more than the control parameters considered above. In general more uniform channel shape and dimensional were found for the mid ranges of the processing parameters. This is represented in the fact the channel dimensions predicted for channel two were closer to those measured than for either of the other two channels.

\subsection{Channel morphology}

Figures 6,8 and 10 show the predicated versus the experimental 3D geometry of channels 1,2 and 3 respectively. These profiles were found to repeat over the length of the channel in the model and experimental results. In each case therefore, the dimensions resulting from a single laser pulse were plotted. The viewing angle of the 3D view was modified to show most of the channel parameters for both the predicted and experimental channels. Figures 7, 9 and 11 show the side view of the same channels. The results represent the laser deposition propagating along the positive $\mathrm{x}$-axis. All axes measurements were taken in $\mu \mathrm{m}$ and the length scale on these figures is the same for direct comparisons. The periodicity and good matching of channel dimensions in these profiles is evident from examination of figures 6 to 11.

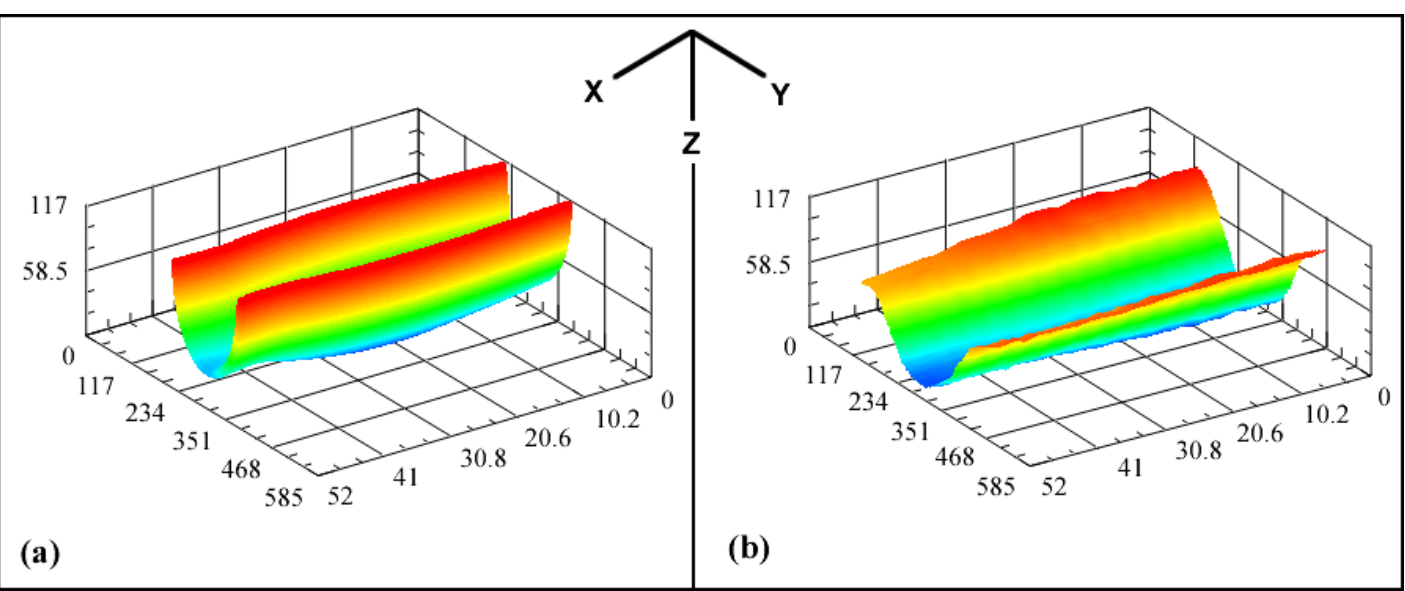

Figure 6: Channel 1, 3D views (a) predicted and (b) experimental scan. (units $\mu \mathrm{m}$ ).

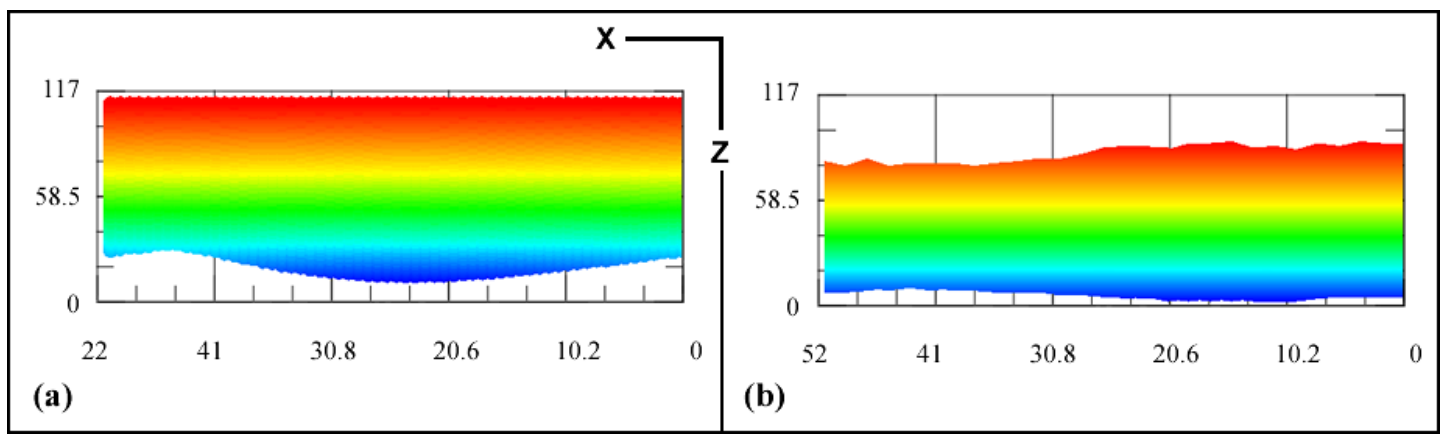

Figure 7: Channel 1, side views (a) predicted and (b) experimental scan. (units $\mu \mathrm{m}$ ). 


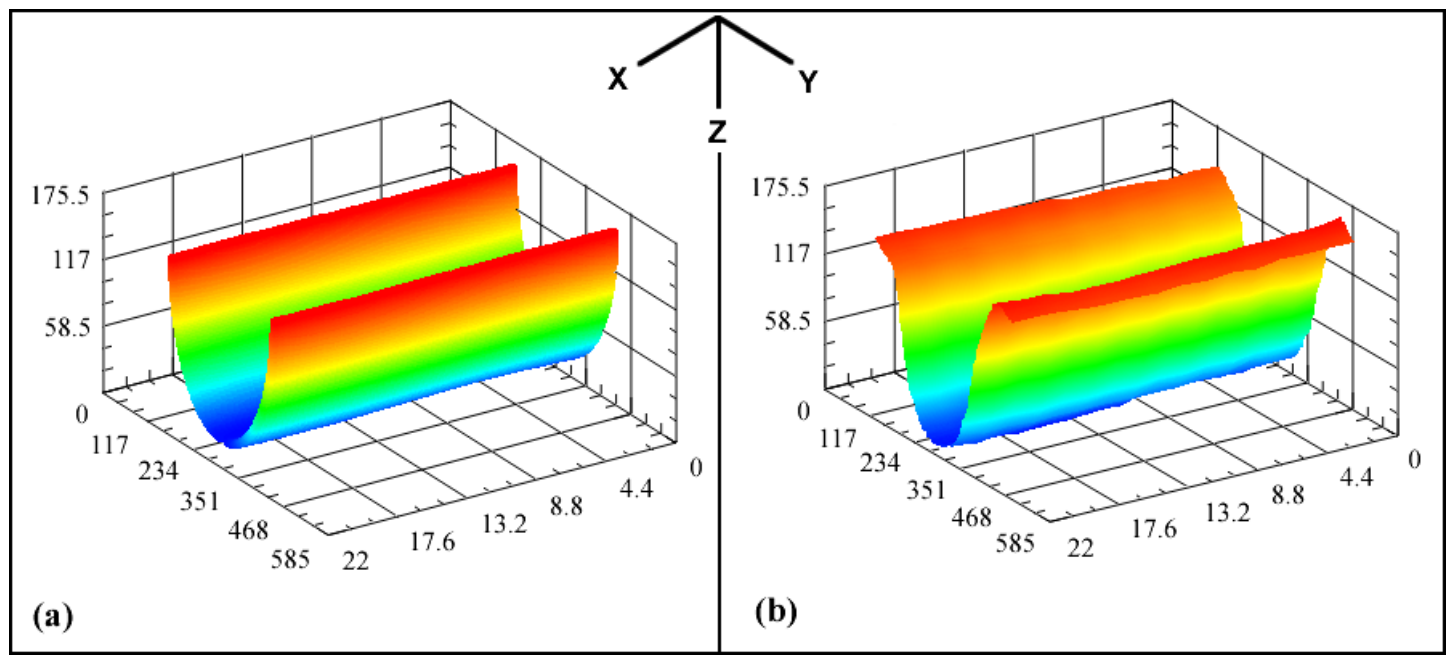

Figure 8: Channel 2, 3D views (a) predicted and (b) experimental scan. (units $\mu \mathrm{m}$ ).

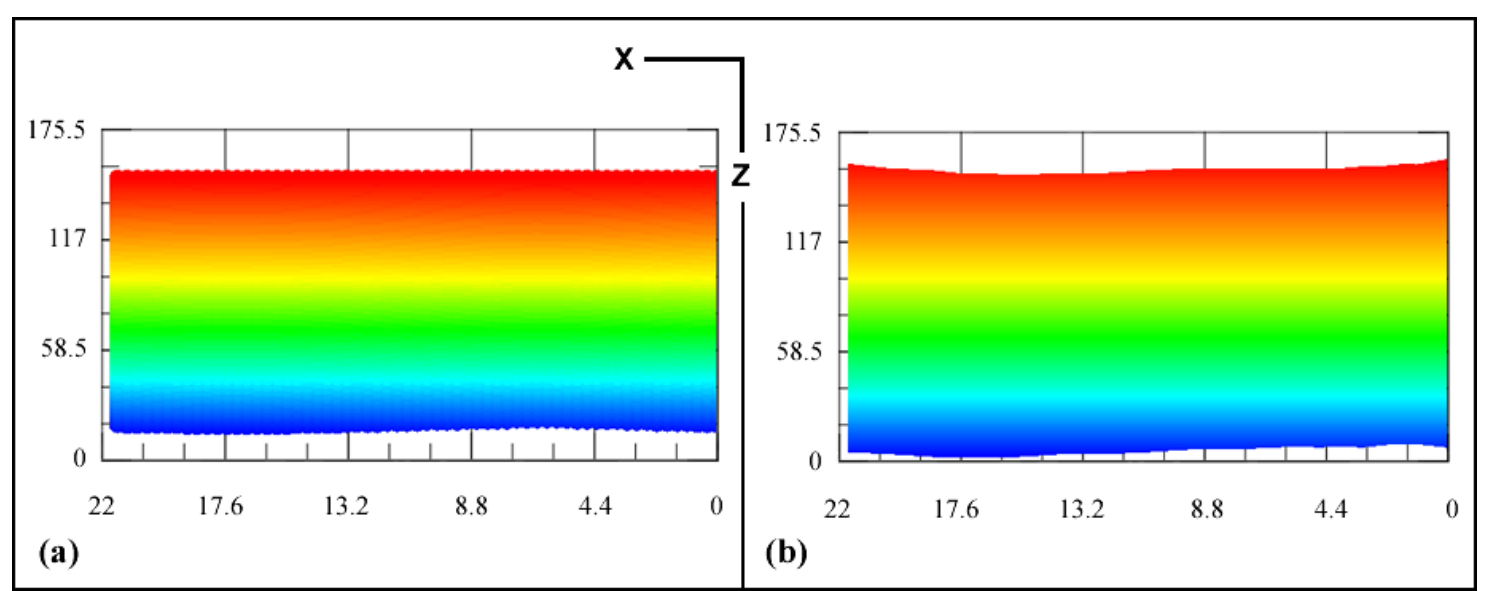

Figure 9: Channel 2, side views (a) predicted and (b) experimental scan. (units $\mu \mathrm{m}$ ).

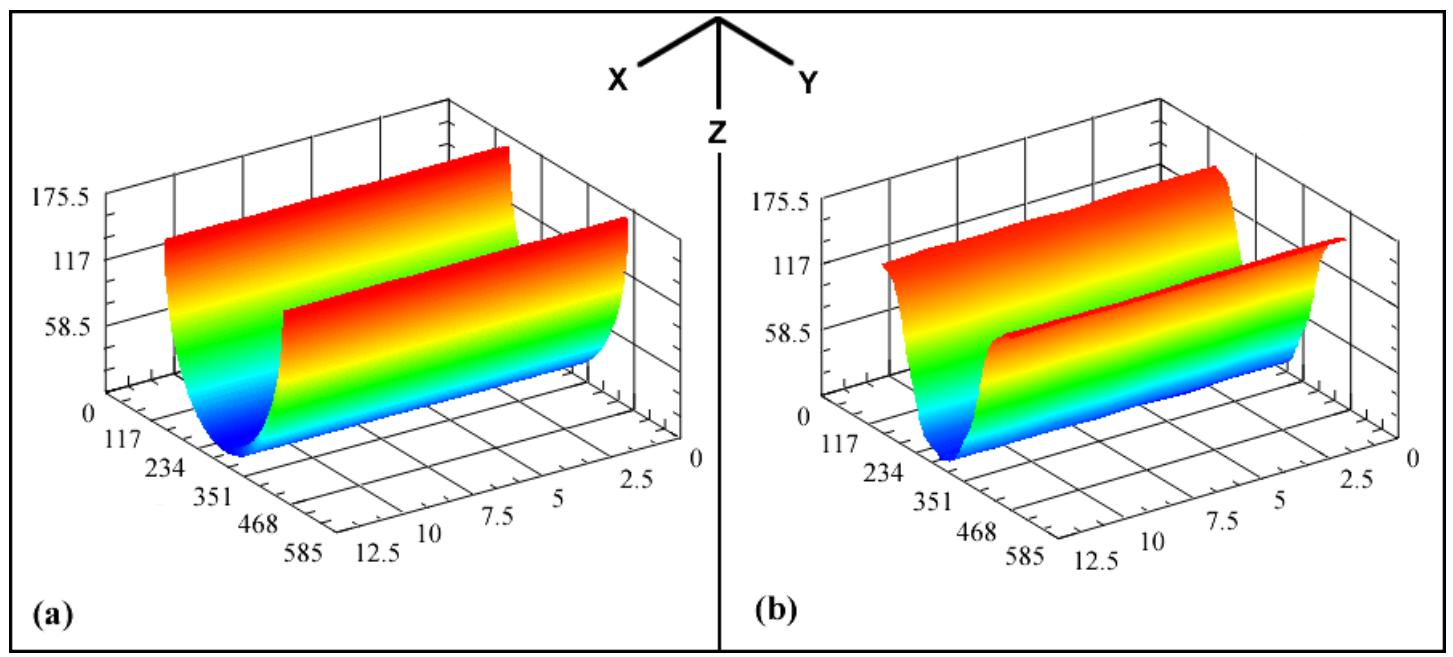

Figure 10: Channel 3, 3D views (a) predicted and (b) experimental scan. (units $\mu \mathrm{m}$ ). 


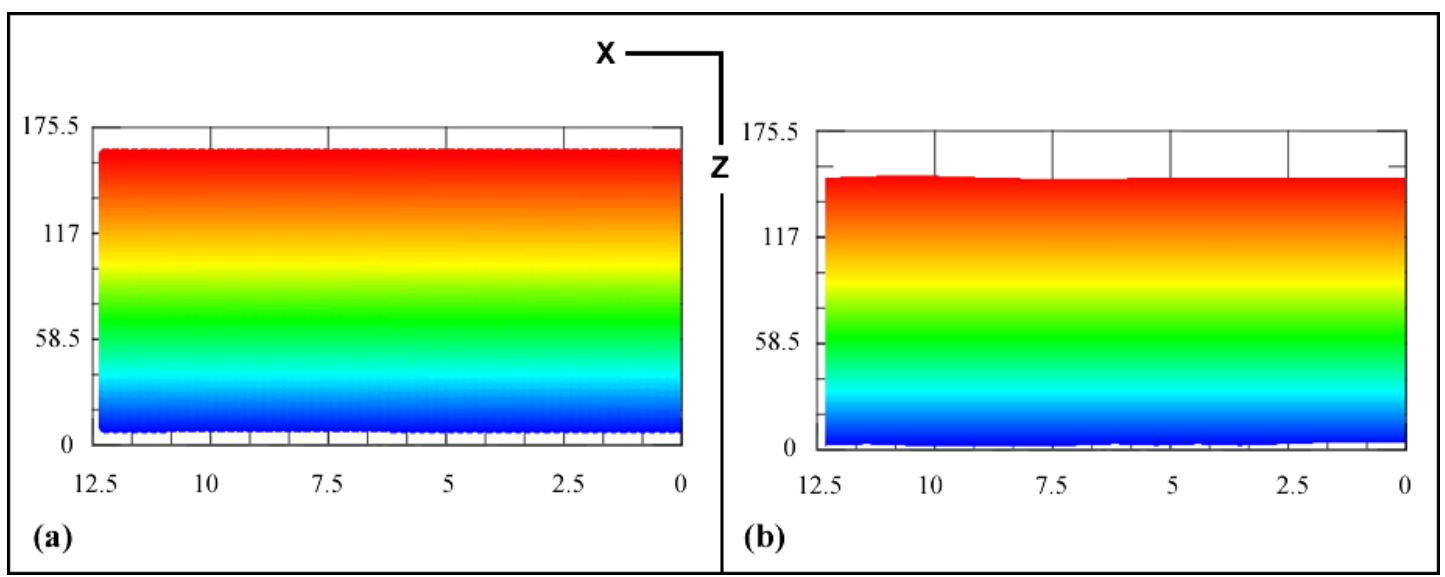

Figure 11: Channel 3, side views (a) predicted and (b) experimental scan. (units $\mu \mathrm{m}$ ).

Figure 12 (a) shows the microscopic image of channel 1 and figure 12 (b) shows a simulated channel length equal to the length in the microscopic image for a direct morphological comparison. From the PRF and U setting used to produce this channel, it was expected that each laser pulse would cover a distance equal to U/PRF $=8.333 / 160 \approx 52 \mu \mathrm{m}$. It can be observed from figure 12 (a) that the channel consisted of an array of helical shapes that were periodic in nature. These shapes were concaved opposite to the laser ablation direction, which was from the left to the right of figure 12 (a). The separation between these shapes was, on average, around $50 \mu \mathrm{m}$. The simulated channel length contains a plot of 22 pulse shapes, and it conforms to the experimental result from the morphological perspective. The width scale of the simulated channel is half the length scale.

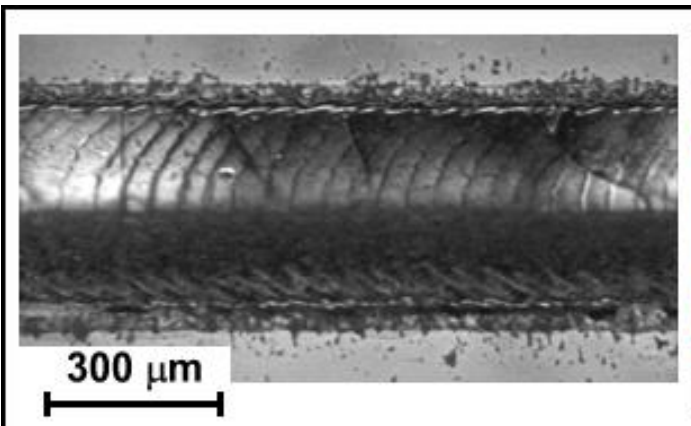

(a)

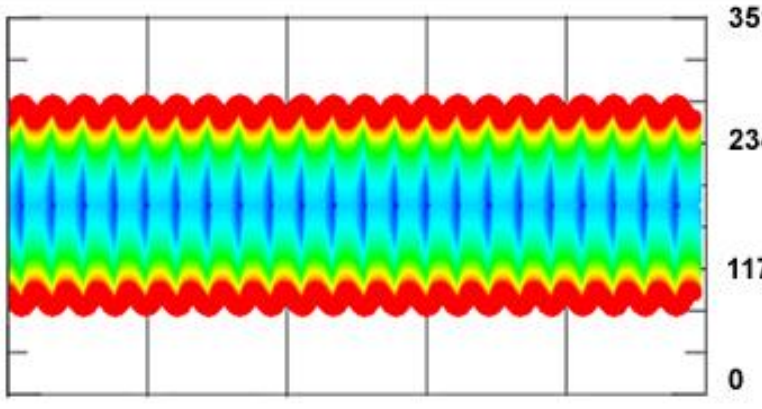

(b)

Figure 12: Morphological comparison of channel 1.

\section{CONCLUSION}

Microchannels were produced using a direct writing single pass laser ablation technique rather than multiple laser passes, as was applied in previous work [8]. Due to the controllability of the profiles fabricated, these microchannels can be designed a priory in order to take advantage of specific channel dimensions in applications such as MEMS and cell growth. Thermal mathematical modelling, with input parameters of material physical properties, laser average power, pulse repetition frequency, pulse width and translational speed, is useful in visualising the expected channel shape and dimensions before channel fabrication. Moreover, 
such results may be helpful in picking the process control parameters, by viewing their effects on the channel geometry. Larger dimensional errors between predicted and experimental results were noted at the extremities of the parameter ranges investigated. However, even in these cases the model was still useful in determining the morphology of the fabricated channels.

\section{References}

[1] W. W. Duley, Laser processing and analysis of materials, Plenum Press, New York, (1982).

[2] N. Bansal and R. Doremus, Handbook of glass properties, Academic Press Inc., Orlando, (1986).

[3] J. O. Isard, Surface reflectivity of strongly absorbing media and calculation of the infrared emissivity of glasses, Infrared Physics, Vol. 20 (1980), 249-256.

[4] L. J. Radziemski and D. A. Cremers, Laser-induced plasmas and applications, Marcel Dekker Inc., New York, (1989).

[5] S. Juodkazis, K. Yamasaki, A. Marcinkevicius, V. Mizeikis, S. Matsuo, H. Misawa and T. Lippert, Microstructuring of silica and polymethylmethacrylate glasses by femtosecond irradiation for MEMS applications, Proc. Mat. Res. Soc. Symp., 2002, B5.25.1-B5.25.6.

[6] K. Miura, J. Qiu, H. Inouye, T. Mitsuyu and K. Hirao, Photowritten optical waveguides in various glasses with ultrashort pulse laser, Appl. Phys. Lett., Vol. 71 (1997), 3329-3331.

[7] S. J. Qin and W. J. Li, Process characterization of fabricating 3D micro channel systems by laser-micromachining, Sensors and Actuators A, 97-98 (2002), 749-757.

[8] S.-C. Wang, C.-Y. Lee and H.-P. Chen, Thermoplastic microchannel fabrication using carbon dioxide laser ablation, Journal of Chromatography A, 1111 (2006), 252-257.

[9] J. M. Dowden, The mathematics of thermal modelling: an introduction to the theory of laser material processing, Chapman and Hall CRC, New York, (2001).

[10] H. S. Carslaw and J. C. Jaeger, Conduction of heat in solids, $2^{\text {nd }}$ edition, Clarendon Press, Oxford, (1959).

[11] D. Collins, Development of laser based surface profilometer using the principle of optical triangulation, MEng thesis, Dublin City University, Dublin, (2005).

[12] Y.-Ill Lee, K. Song and J. Sneddon, Laser-induced breakdown spectrometry, Nova Science Publishers, Inc., New York, (2000).

[13] A. Issa, D. Brabazon and S. Hashmi, Process modelling of micro channels production using $\mathrm{CO}_{2}$ laser in silica glass using RSM, Proc. of the Int. Conf. on Advances in Materials Processing and Technologies (AMPT), Las Vegas, 2006, NII6, 103, 1-5.

[14] A. Issa, D. Brabazon and S. Hashmi. Improvement of lime soda glass laser microfabrication processing parameters using design of experiments, in C. Bocking, D. Jacobson and A. Rennie (eds). Proc. $7^{\text {th }}$ National Conference on Rapid Design, Prototyping and Manufacturing (RDPM), Buckinghamshire-UK, 2006, 9-20. 